\title{
Frontiers in Polymer Chemistry
}

\author{
A. Dieter Schlüter*
}

\begin{abstract}
The article shows how the initial concept of Staudinger on linear macromolecules was expanded topologically by increasing the cross-section diameter of polymer chains and by introducing sheet polymers with planar rather than the commonly known linear repeat units. The two concrete projects addressed are the synthesis of dendronized and of two-dimensional polymers. It is explained how these novel macromolecules were achieved and which obstacles had to be overcome but also where these frontiers in polymer chemistry might lead to new insights in polymer science in general and novel applications in particular. The article also provides insights into analytical issues because both target macromolecules are in an extraordinarily high molar mass range and contrast/sensitivity issues can turn rather serious in particular for the two-dimensional polymers.
\end{abstract}

Keywords: Dendronized polymers · Two-dimensional polymers

\section{Introduction}

In 1912, thus one hundred and one years ago, Hermann Staudinger commenced his professorship at ETH Zürich and soon thereafter published groundbreaking papers on his perception of macromolecules. [1] Despite serious disputes with those of his colleagues who did not believe that there are molecules larger than a crystallographic unit cell, his experimentally carefully substantiated view of the existence of long linear chain molecules (rather than colloidal assemblies of small molecules) became increasingly more accepted worldwide, and ultimately proved indeed to be correct. ${ }^{[2]}$ Nowadays, macromolecules, also referred to as polymers - or more mundanely as plastic, and the products made with them can be found in almost every aspect of life ranging from nutrition to healthcare and from transportation to clothing [World plastics production 2012: 265 million tonnes. http:// www.plasticseurope.org], and are of an enormous economic importance. Because of the huge impact of linear (and crosslinked) macromolecules to all of us, there has been an intense research effort both in industry and academia aiming at ever better synthesis procedures, structurally new polymers and novel processing schemes leading to both new fields of application as well as replacement of other materials (e.g. metals) in known applications. The improvements in classical and supported metallocene catalyst systems shall serve as a first example here. ${ }^{[3]}$ They provided

\footnotetext{
${ }^{*}$ Correspondence: Prof. Dr. A. D. Schlüter

Laboratory of Polymer Chemistry

Department of Materials

ETH Zurich, $\mathrm{HCl} \mathrm{J} 541$

Wolfgang Pauli Strasse 10, CH-8093 Zürich.

E-mail: ads@mat.ethz.ch
}

access to extremely highly isotactic polypropylene (it-PP), which is an important engineering plastic particularly in the automotive industry. Note that today's plastics make up 50 percent of the volume of new cars but only 10 percent of the weight. ${ }^{[4]}$ But also tuning the molar mass and the molar distribution of many vinyl polymers under rather robust conditions is not so much an issue anymore as it was some 25 years back. Controlled radical polymerization procedures have opened up a new avenue into this field and to some degree replaced the experimentally quite demanding anionic polymerizations. ${ }^{[5]}$ Of the many polymers with novel structures that were synthesized in the past few decades, polyfluorenes (PF) deserve some attention. ${ }^{[6]}$ They were made available through recently developed transition metal-mediated cross-coupling protocols, one of which is Suzuki polycondensation. [7] PFs are typically used in form of copolymers e.g. as active components in organic light emitting diodes (OLED). Finally, examples of novel processing schemes are, for instance, the development of spinning fibers from liquid-crystalline (lyotropic) solutions of the rigid macromolecule poly(para-phenylene therephthalamide) (aramids) from sulphuric acid[8] and socalled gel-spinning of ultra-high molecular weight $(>3.106 \mathrm{~g} / \mathrm{mol})$ polyethylene ${ }^{[9]}$ that have a stiffness and strength 5-10 times that of steel on an equal weight basis. These fibers have found numerous applications in the construction, protection, sport and medical industries.

Having this impressive triumph of linear macromolecules laid out in front of us, we wondered whether there was still room for progress from the fundamental side. Were there challenges that had not been looked into and which promised to lead to useful developments once tackled?
After intense thinking and brain-storming with my co-workers and colleagues two research targets were in fact identified and considered worth pursuing: 'thick' linear macromolecules and two-dimensional (sheet-like) polymers (2DP). This short review article tries to introduce the reader to the merits of these two targets and where we see their potential importance. It begins with a few introductory comments, while the major part of the article will go more intensely into thickness and dimensionality matters in connection with polymers.

Linear polymer chains are available with various different main chain structures as well as various different lateral substituents. Examples are poly(hydroxyl acrylate), 1, and polystyrene, $\mathbf{2}$, which are important components in superabsorbent hydrogels and for packaging applications, respectively (Fig. 1). The cross-section diameters (thickness) of their backbones are increased by the lateral substituents, which are hydroxyethylester for $\mathbf{1}$ and phenyl for 2 . While these substituents naturally affect somewhat backbone properties, such as persistence length (a measure for chain stiffness) and entanglement length (describing the average chain length between entanglements), in a first approximation their impact on main chain crosssection diameter is small, irrespective of the fact that such a diameter is difficult to define anyway. This becomes evident when comparing these polymers with the linear polymethylmethacrylate (PMMA) derivative $3,{ }^{[11]}$ in which the 'tiny' methyl group of the ester is replaced by a large, regularly branched (dendritic) substituent (Fig. 1). Such a huge substituent at every other backbone $\mathrm{C}$-atom increases the cross-section diameter enormously and by this, changes the polymer fundamentally. Polymer $\mathbf{3}$ is not a PMMA anymore with a property profile making it the famous 
commercial product Plexiglass ${ }^{\mathrm{R}}$; rather it is the lateral substituents in $\mathbf{3}$ which control everything and downgrade the backbone from a major player, particularly in terms of entanglement formation, to a simple scaffold just holding all this mass together. The backbone cannot undergo the slightest conformational change anymore without 'permission' from the voluminous mass surrounding it. Clearly something new has been created. All of a sudden one can ask, for example, whether small compounds such as solvent molecules can be put into a polymer main chain (a stupid question in regard to $\mathbf{1}$ and $\mathbf{2}$ ), whether the cross-section of a main chain is responsive to external stimuli or whether such macromolecules are molecular objects that can be rolled across solid substrates. This may suffice for a moment to frame what is meant by thick polymer chains and where the importance of this aspect may lie.

The second target of our group's research efforts aims at a dimensional extension of Staudinger's so successful concept of macromolecules. He viewed macromolecules as long, linear, covalently bonded arrays of repeat units (RU) and, thus, defined a polymer chain by the chemical structure of a RU, the number of RUs and the two end groups that terminate the chain at both ends. This concept has been expanded over the decades to branched and cyclic polymers as well as various forms of copolymers, which however all have topologically linear RUs (except for the branching points). Looking at macromolecules under dimensionality aspects, we wondered whether it would not be possible to create representatives with topologically planar RUs. Such RUs would give polymers a second dimension and would free macromolecules from being restricted to topologically one-dimensional (linear) geometry. The term macromolecule would then also include monomolecular sheetlike objects, the entire structure of which is a tessellated array of RUs terminated by an 'infinite' number of end groups positioned at the sheet circumference. Fig. 2 illustrates the difference between a classical Staudinger polymer and a two-dimensional polymer (2DP) as we see it.

Because of the many insightful investigations carried out on ultrathin polymer films, it is important to avoid confusion with this particular class of materials. We therefore emphasize again that the second target of our studies does not aim at yet another ultrathin polymer film but rather at solving the fundamental synthetic problem associated with the generation of monolayer sheets solely consisting of topologically planar RUs. Fig. 2 pinpoints the difference. While currently known ultrathin films cannot be described by RUs and often even have a completely disordered, randomly
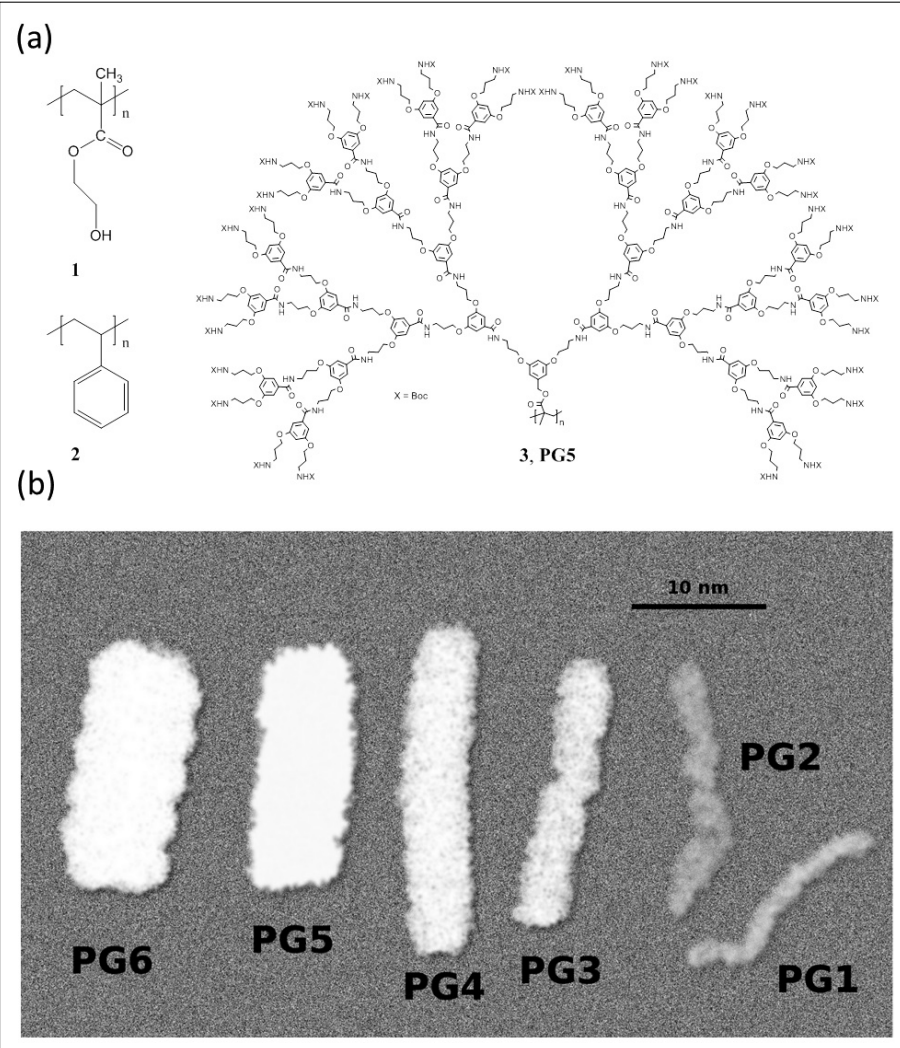

Fig. 1. (a) Chemical structures of typical linear polymers with low cross-section diameter, $\mathbf{1}$ and $\mathbf{2}$, and a dendronized polymer (DP) 3 (PG5) with a huge regularly branched substituent at every other backbone carbon atom resulting in a 'thick' polymer with an unprecedentedly large cross-section diameter. (b) Results of molecular dynamics simulations of six dendronized polymers differing by the dendron generation $g$ from $g=1-6$ (PG1PG6). Courtesy of Prof. C. Aléman and Dr. Oscar Bertran, TU Barcelona, Spain. See ref. [10].

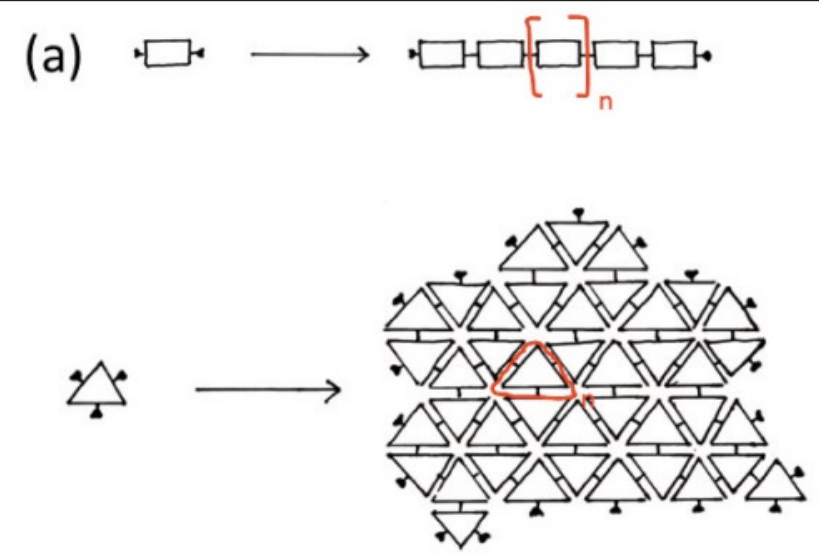

(b)

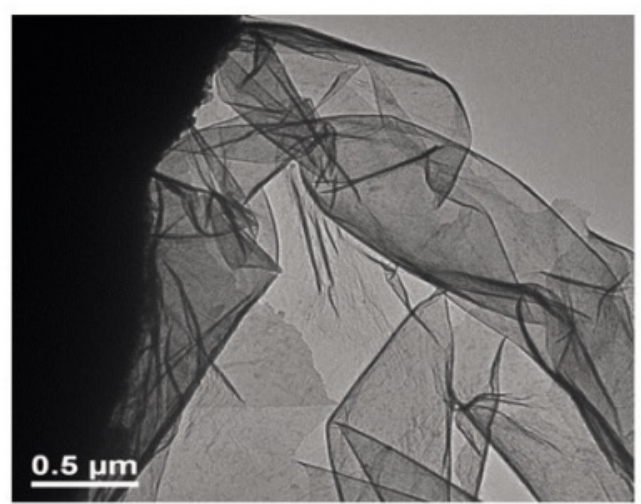

(c)

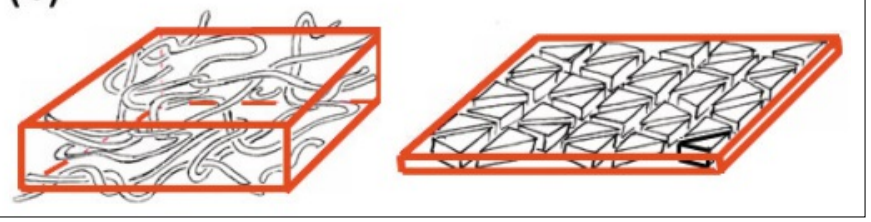

Fig. 2. (a) Illustration of the difference between a linear (top) and a twodimensional polymer (2DP) (bottom).

While the former has linearly connecting RUs, the latter has laterally connecting RUs resulting in a periodic monolayer sheet with internally tessellated (periodic) molecular structure. (b) TEM micrograph of a 2DP. (c) Cartoon representations of a conventional ultrathin polymer film and a 2DP. While the mechanical strength of the former rests upon the chemical and physical netpoints (cross-links and entanglements, respectively), the latter forms a mechanically coherent entity because of its regularly tessellated internal structure consisting of planar RUs. 
cross-linked internal structure, ${ }^{[12]}$ the sheets aimed at here are in principle characterized by a periodic array of covalent cross-links over their entire expanse. Such a complex target raises, of course, the point why one should go through all the effort with devising synthetic strategies and analyzing (intrinsically low contrast) structures when slightly thicker films with no internal order can easily be accessed? Or in other words: What are 2DP good for? The answer to this question is twofold. On the one hand, these unique polymers are expected to add to the fundamental understanding of polymer science and, on the other, they will lead to whole bouquet of novel applications. The fundamental understanding would be advanced because the entire polymer physics was developed to describe and predict properties of linear chains and - with regular systems at hand can now be reasonably expanded and adjusted to polymers of higher dimensionality, the sheets. Regarding the applications, 2DP might prove useful as nano-scale membranes (where they offer monodisperse holes), for sensing, catalysis, molecular landscaping in general and all other aspects that leverage their very particular molecular organization.

\section{Thick Polymers and Cylindrical Molecular Objects}

The divergent growth concept, as first reported by Vögtle, [13] is a powerful synthetic measure to increase the size of any molecule that carries at least a few functional groups to start the growth from. This has impressively been shown by Tomalia for dendrimers, which are more or less spherical macromolecules, the diameter of which depends on the number of consecutive divergent growth steps.[14] Their divergent synthesis starts from a small oligofunctional core molecule to which branches are added step-by-step, generation $(g)$ by generation. A bench mark synthesis in terms of size achieved and number of growth steps is Majoral's dendrimer with twelve branching points and thus $g$ $=12 \cdot{ }^{[15]}$ This tool was available when our group in 1994 started to do first steps towards systematically thickening polymer chains. ${ }^{[16]}$ Fig. 3a shows the outline of the applied protocol. It starts from a first generation polymer $(g=1$; PG1) whose molar mass had precisely been determined by light scattering and gave the equivalent of 10600 RUs. PG1 carries two protected terminal amine groups, the (Boc-) protection groups, which needed to be completely removed under acidic conditions before the resulting ammonium-terminated intermediate (not shown) could be brought to reaction with an excess of the dendronization

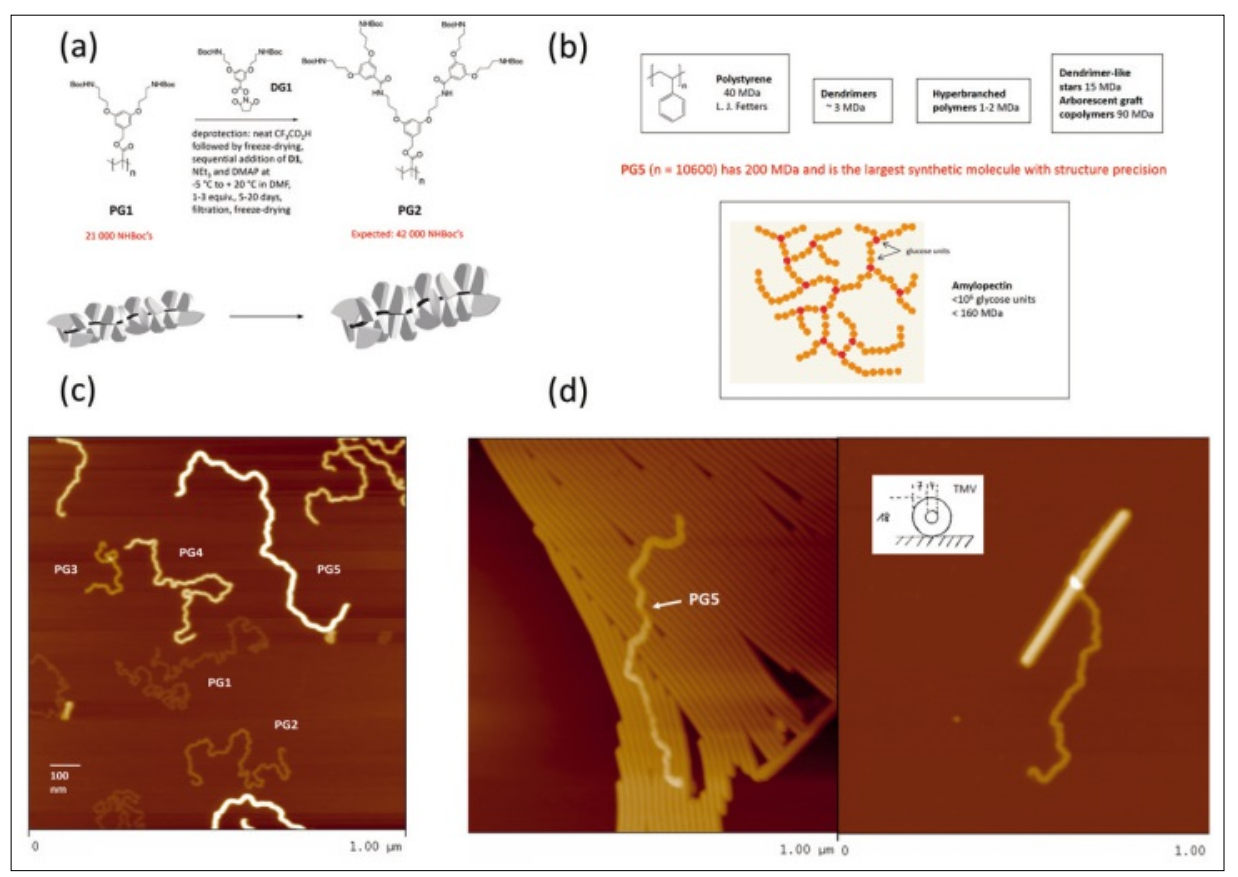

Fig. 3. (a) Recursive chemical equation explaining how to synthesize DPs with ever increasing $g$. (b) Comparison of the molar mass of PG5 with $10600 \mathrm{RUs}$ with other synthetic polymers as well as the biological macromolecule amylopectin. (c) AFM height image of co-prepared PG1-PG5 chains to visualize the increased chain stiffness (decreasing undulations) and increased chain cross-section dimensions (thickness) with increasing g. (d) AFM height images of a single PG5 chain on an array of Tobacco Mosaic Virus (TMV) and of a single chain embracing a single TMV on mica.

agent DG1. After supposedly each terminal amine functional group has reacted with DG1, the $g=2$ DP PG2 is obtained. This procedure can then in principle be repeated over and over again until the DP with desired $g$ is reached. In the case mentioned above, Dr. Baozhong Zhang, a senior scientist in our group, carried the sequence through to PG5 which - after careful defect quantification ${ }^{[17]}$ - had a molar mass of approximately $200 \mathrm{MDa} .^{[11]}$ In the last step, when going from PG4 to PG5, the mass increase amounted to $100 \mathrm{MDa}$. This increase, which proceeds in a controlled fashion, is the largest mass that has ever been generated in a single reaction step to give a structurally defined, covalent molecule and is more than any polymerization reaction has so far afforded. Also in terms of total molar mass, this reaction is remarkable and shows the enormous potential of divergent growth. With its molar mass of 200 MDa, PG5 not only by far exceeds all synthesized macromolecules (Fig. 3b) but also surpasses most biological macromolecules. Amylopectin, the storage form of glucose, ranges in the same mass category and only the DNAs, which can contain millions of nucleotides, are still much higher in molar mass. The AFM height image in Fig. 3c shows semi-quantitatively that the DPs become increasingly more rigid and also thicker with increasing $g$.

While an average molar mass of 200 MDa for a single molecule may sound impressive, one needs to ask whether the creation of such a mass has any meaning because it leads into novel, unexplored scientific terrain. As the AFM images in Fig. 3 suggest, DPs with increasing $g$ turn more and more into molecular objects to which an interior and an envelope can be assigned. However, increasing of $g$ cannot be continued forever: Mass increase per divergent growth step is exponential while increase in cross-section span is linear. ${ }^{[18,19]}$ Thus, there must be a point where all branches are densely packed around the backbone and further growth can only be achieved at the expense of reduced structure perfection. For packing reasons not all terminal amine groups that could react with a dendronization agent have the space available anymore to actually do so. It is thus noted that DPs have two different $g$ regimes. They need to be differentiated, in order to understand what DPs actually are, what thick molecular object actually means, in terms of structure perfection but also in terms of property profile. For the first regime $g<g_{\max }$ applies and for the second $g \geq g_{\max }$. The straight (black) line in Fig. 4c marks the maximum cross-section span as it develops with $g$, while the green line is the span resulting from pure density considerations (assuming in a first approximation a density $\left.\rho \sim 1.1 \mathrm{~g} / \mathrm{cm}^{3}\right)$. ${ }^{[19]}$ $g_{\max }$ is at their intersection and thus at approximately $g=6.5$ (for this particular molecular structure). Below this point both lines enclose an area which means that DPs below $g_{\max }$ should be responsive to external 
stimuli. ${ }^{20]}$ Their maximum cross-section is not yet reached in the dry state and their interior has capacity to accommodate solvent or guest molecules (such as drugs) to the point that the branch work is fully stretched out and reaches the black line. On the contrary, at and beyond $g_{\max }$, DPs should (ideally) not be responsive anymore with interesting consequences in terms of properties and potential applications. But now let's first concentrate on the $g<g_{\max }$ regime.

Below $g$ the branch work around the backbone of DPs has capacity to take up solvent and other molecules. ${ }^{[21]}$ This is a unique property of DPs and sets them apart from common linear polymers, the backbones of which have no internal volume. This useable volume of DPs qualifies them as molecular containers which can in principle be used to selectively transport cargo from one side of a membrane to another, reminiscent of DNA translocation through nanopores. ${ }^{[22]}$ The uptake is driven by the osmotic pressure or specific interactions between guest molecules and the DP host. DPs can also squeeze out their guest molecules under certain conditions and thus force the DP chains to precipitate from solution (loss of osmotic stabilization). This squeezing out, which is an important process e.g. in regard to unloading of cargo, can be achieved by temperature changes if the dendron/dendron and dendron/guest molecule interactions are carefully chosen.[20] The property of temperature-dependent loading and unloading and its direct impact on persistence lengths may lead to new materials based on slightly cross-linked DPs. Such networks may have an extraordinary temperature dependence of their elastic moduli. Because for $g<g_{\text {max }}$ the backbone is not in its fully stretched conformation, the contour length is subject to change. [23] Changes can be induced by external stimuli such as $\mathrm{pH}$ value. If one uses DPs with unprotected terminal groups the $\mathrm{pH}$ value of the surrounding aqueous medium controls the level of charging. The lower the $\mathrm{pH}$ the more effective charges will be placed on the DP resulting in a stretching of the backbone by charge repulsion. If the $\mathrm{pH}$ is increased, however, the number of effective charges on the DP is reduced allowing its backbone to contract for entropic reasons. Such changes in contour lengths have been determined by AFM pulling experiments. It was found that contractions by $20 \%$ are within reach and that the forces associated with this contraction are in the range of 1 $\mathrm{nN}$. ${ }^{[23]}$ This in principle qualifies DPs for applications in molecular machines, e.g. to lift a weight that is fixed to one end of a DP chain. Fig. 5a displays cartoons describing these properties of $g<g_{\max }$ DPs.

While the exploration of the $g<g_{\max }$ regime is well underway and has already led

\section{(a)}

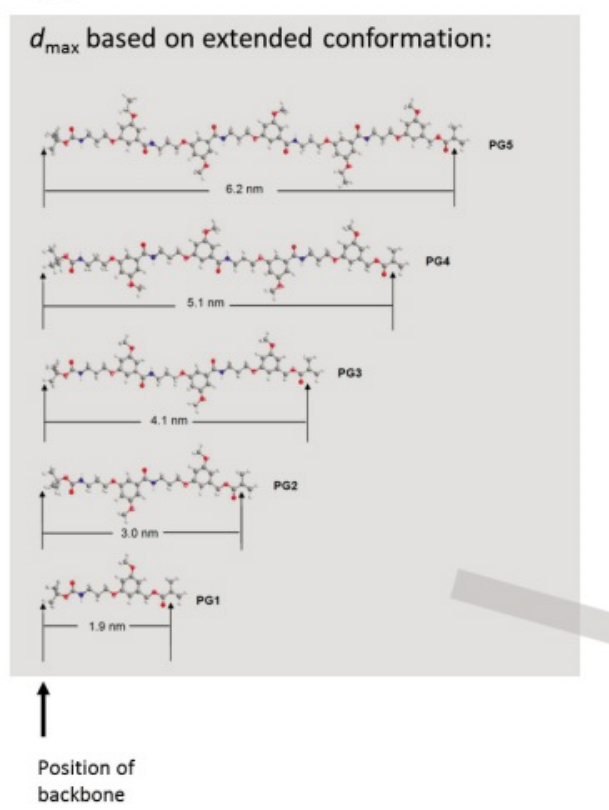

(b)

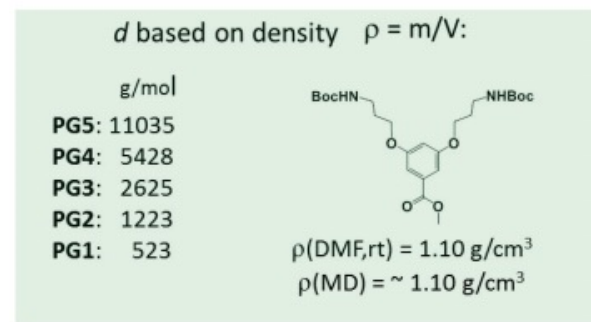

(c)

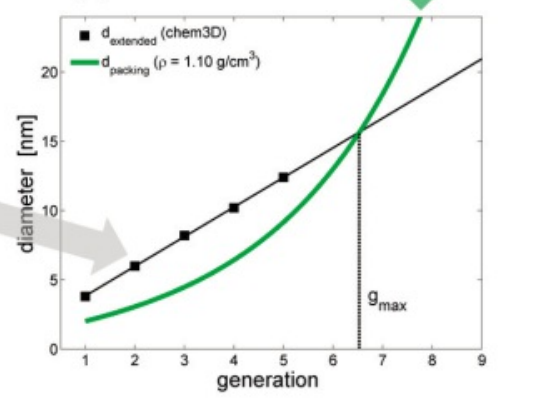

Fig. 4. (a) ChemDraw structures of parts of dendrons of generation $g$ emanating from the backbone in a stretched conformation and thus defining the corresponding DPs maximum crosssection span (multiplied by factor 2). (b) Molar masses of the RUs of PG1-PG5 and the diameter resulting from such masses based on density (from measurements with the shown model compound and molecular dynamics (MD) simulation). (c) The $g$ dependencies resulting from (a) and (b) and their intersection at $g=6.5$ which marks $g_{\max }$. to fascinating findings there is no concrete knowledge yet about the $g>g_{\max }$ regime. The reason for this is lack of experimental availability. Only very recently in our group the doctoral student Hao Yu reached and surpassed $g_{\max }$ DPs synthetically and one needs to wait until the first publica- tions aiming at their properties become available. Importantly, a combined analyti$\mathrm{cal} /$ theoretical tool has already been develdelicate range. ${ }^{[24]}$ Fig. 5b displays a dream of the author in this regard. As mentioned above, at and beyond $g_{\max }$ DPs should have oped to try to quantify defects also in this

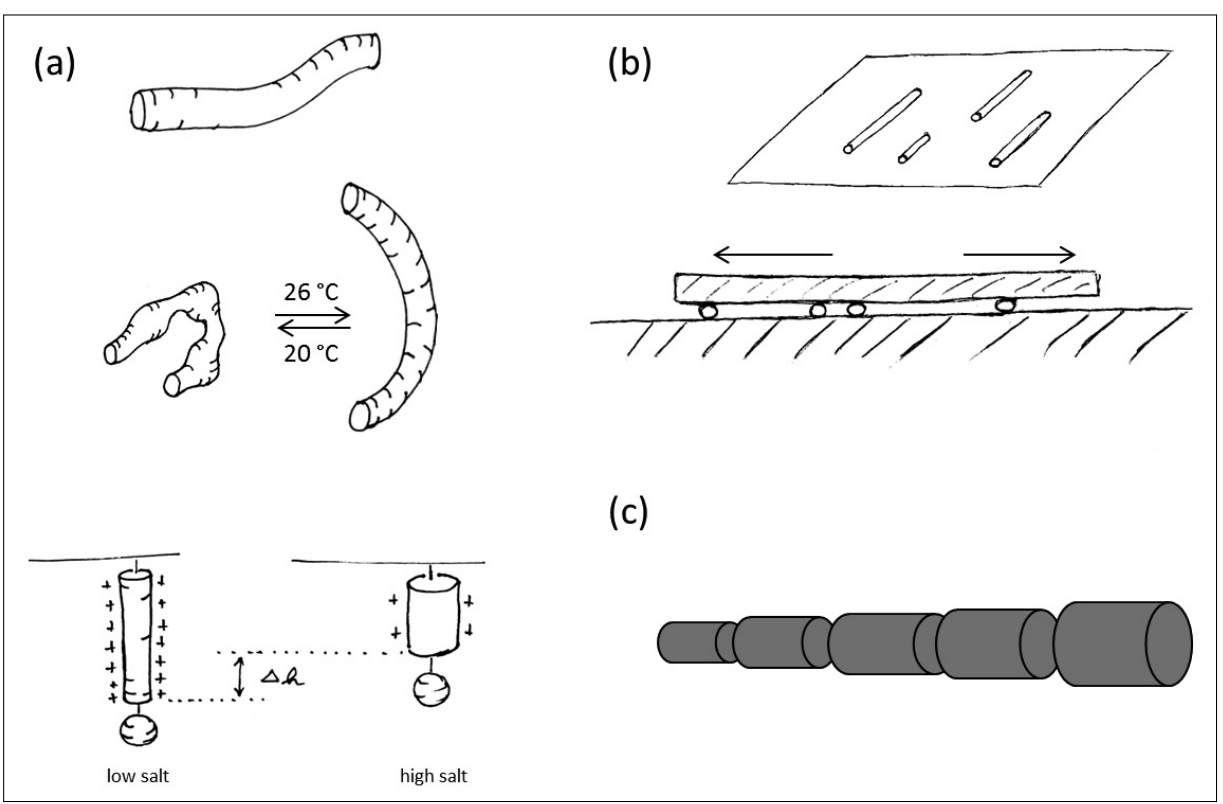

Fig. 5. (a) Typical responsiveness in the $g<g_{\max }$ regime: (top) loading capacity of the cylindrical objects as defined by unused volume compared to fully stretched structure; (middle) strong response of temperature on backbone stiffness for DPs in theta solvents; (bottom) charged DPs as molecular machines which, depending on salt concentration change can contract or elongate. (b,c) Two (future) examples for the $g>g_{\text {max }}$ regime: Rolling of DPs to be used for anisotropic lubrication and creation of a homologous series of single molecule colloidal particles with quantized diameter difference of $\sim 1 \mathrm{~nm}$ between next neighbor DPs. 
a densely packed dendritic branch work surrounding their backbone, ideally not allowing for molecules to penetrate into their interior because of a densely packed structure. This dense packing results in molecular objects, which are characterized by large molecules whose shape is independent of the environment the objects are exposed to. If this picture holds true, the adsorptive forces DPs are exposed to when placed on solid substrates should be small because of the small contact area. Ideally one can therefore expect the objects to be rollable on the substrate, similar to as it was shown for carbon nanotubes. ${ }^{[25]}$ This would be an attractive feature because it should open the way to molecular lubrication and, if the DPs can be oriented, even to anisotropic lubrication (Fig. 5b). The $g$ $>g_{\max }$ regime however is expected to offer even more. As mentioned, the first DPs with $g$ beyond $g_{\max }$ have been synthesized. This is to be considered the first step towards molecular colloidal particles. Because there are countless procedures leading to colloidal particles based on polymers, ${ }^{[26]}$ it is important to consider the features DPbased particles would be offering. In the first place this is their cylindrical shape combined with monodisperse diameters. Everybody who has experience in the selfassembly of particles will appreciate it if the particles used are strictly monodisperse and size segregations are avoided to begin with. In addition to this, entire homologous series would be available (Fig. 5c), next neighbor members of which differ by approximately $1 \mathrm{~nm}$ in diameter only. This allows for an extreme fine tuning in terms of supramolecular aggregates compared to what is possible these days with conventional colloids. ${ }^{[26]}$ Finally, it should be mentioned that the internal segment distribution of colloidal DP particles is much better known than in any other cross-linked nanoparticles. While the precise spatial position of a certain branch point within a given particle can of course not be foreseen, the probability distribution function can well be established. This is an unheard structure precision for colloidal particles and will be of value for future applications.

\section{Two-dimensional Polymers (2DP)}

The term 2DP has often been used in the literature but in a less strict sense than the one advanced by our laboratory, which - as mentioned above - is based on two ingredients, the concept of RUs originating from Staudinger and the monolayer nature. This short highlight cannot possibly offer a comprehensive treatment of the available literature to this rather complex matter and the reader is therefore referred to a 2009 review article by Sakamoto et al. ${ }^{[12]} \mathrm{A}$ very recent case of a differing definition shall be mentioned, however, to illustrate how important it is to find a consensus. Colson and Dichtel[27] suggested in a 2013 publication to not only consider macromolecules as 2DP that meet our definition but to also include "all covalently linked networks of monomers with periodic bonding in two orthogonal directions" irrespective of whether they have been isolated in "layered crystals, multilayer or monolayer films". This view was supported by comparing to linear polymers for which the authors correctly stated that linear (one-dimensional) polymers remain as such whether they are found in the bulk, dissolved in solution, or isolated as individual macromolecules. What this comparison however lacks is the important aspect that for linear polymers it was proven countless times that, irrespective of whether they are in bulk, solution or as individualized entities, they are in fact clearly identifiable individual chains. This was achieved, e.g., by dissolving a (noncross-linked) bulk polymer into a solvent in which the then untied chains enjoy their conformational freedom. The same has not normally been achieved for synthetic layered crystals though. ${ }^{28]}$ In other words, to consider any layered material a 2DP misses the point in the same way that graphite differs from graphene, the prototypical 2DP provided by Nature. Graphite would not normally be considered a 2DP. A discussion on this seemingly subtle discrepancy between definitions was chosen as the entrée for this small chapter because it is in fact not subtle at all but rather touches the very heart of what a 2DP is and what not, and is therefore believed to be instructive to a reader not so familiar with this matter.

While there may be many synthetic paths to 2DP, we concentrated on approaches where specially designed monomers were pre-oriented in two dimensions prior to being connected with one another in a regular fashion. Pre-orientation can be achieved, e.g. in liquid crystalline phases, at an air/water interface and in layered single crystals. Our laboratory pursued and is still pursuing the last two approaches. Because structure characterization is particularly complicated with monolayers, the interfacial experiments have not yet reached the maturity[29] of the ones based on layered single crystals. ${ }^{[30]}$ This is why this space-limited article concentrates exclusively on a recently published, first realization of a 2DP based on photopolymerization within a monomer crystal followed by exfoliation of the resulting crystal composed of 2DPs into individual sheets. This selection does therefore not reflect a superiority of one of the approaches over the other.

Fig. 6 shows important intermediate steps towards the first 2DP. In chart (a) the monomer structure is given, which combines a collection of important features. First of all it is shape-persistent, which means its conformational space is reduced, a feature that can facilitate crystallization. Also it contains three anthracene units and six acetylene units (marked in red) that are held together by triphenylene bridges. Both anthracenes and acetylenes are photosensitive groups while the rest of the monomer should be photostable at the wavelength used for polymerization later on. Chart (a) also depicts that the monomers in their hexagonally shaped single crystals are arranged up and down in an alternating sequence, whereby - and this is key! - each anthracene of one monomer faces exactly one acetylene unit of the neighboring monomer such that the acetylene is positioned right across the anthracene's 9,10-positions. These positions are prone to undergo cycloaddition reactions with $2 \pi$ - or $4 \pi$-systems if the latter observe the so-called Schmidt distance, [31] which in essence means that the orbitals of both components need to have a minimum overlap to bring the reaction about. Chart (b) illustrates a major breakthrough that all those involved in the group's 2DP project, including Priv. Doz. Dr. Junji Sakamoto and the doctoral student Patrick Kissel celebrated. This breakthrough was not associated with lots of glamour and pomp but rather with a simple solubility difference. As soon as monomer single crystals on a glass plate were reached by a spreading solvent front, they behaved as any single crystal suddenly surrounded by solvent would do: they dissolved. If, however, the single crystal prior to this experiment was photoirradiated at a wavelength to bring about the cycloaddition reaction between the opposing reactive units of neighboring monomers, the crystals remained intact! Thus, something fundamental must have happened with the monomers that prevented them from going into solution. Obviously we suspected that the desired cycloaddition may have happened, which has the potential to convert the entire monomer layers within the crystal into polymer layers. At this point a bit of care is appropriate. The monomer crystals were in the size range of $10-100 \mu \mathrm{m}$ which may be considered relatively small. In terms of the number of monomers being present in each layer ranging from one end of the crystal to the other, however, they were huge. Such a span easily involves 10'000-100'000 monomers. What now is the significance of this number? Assume there is a positional change between next neighbor monomers resulting from the chemical reaction between them. Even if this change is tiny it will add up to a sizeable quantity for a huge number of monomers, which results in formation of small cracks or phase bound- 
aries. This phase change will terminate growth! Thus, it cannot be automatically expected that the 2DP monolayer sheets obtained from such an approach have the same size as the crystals from which they are generated. Also it would not be clear from the very beginning whether crystal geometry (here hexagonal) will actually translate into sheet geometry. After this somewhat cautioning comment it is time to explain what happened when the polymerized crystals were subjected to exfoliation procedures, that is to procedures in which the crystals are exposed to intercalating solvents. ${ }^{[32]}$ Such solvents creep in between the layers, swell the crystals and eventually disintegrate them. Initially sheet packages are obtained which then further exfoliate all the way down to single sheets (presuming sheets are not interconnected through defects). Chart (c) of Fig. 6 shows the process schematically. In fact, monomer crystal could be exfoliated into packages as well as single sheets. The former were used for analysis by transmission electron microscopy (TEM) and electron diffraction (ED) (chart d) and found to be in excellent agreement with the proposed structure of a 2DP. The final breakthrough was achieved by atomic force microscopy (AFM) analysis which gave the expected height of a single sheet for the latter (not shown). As expected, Raman spectroscopy revealed the molecular structure, thus, removing last doubts. In this particular case, the sheets of 2DPs were not yet spanned over holes but must have a considerable mechanical stability because they survived the stressful exfoliation as well as filtration steps through Lacey carbon grids requiring them to crumple and fold up and expand again.

Now that the first case of a 2DP is established, can one lean back and relax? Certainly not! The proof of principle was achieved, yes, but nothing more. Countless issues still have to be addressed, which sketchily include: to create broader structural basis both for the single crystal as well as the interface approach; to facilitate monomer synthesis; to develop large-scale monomer syntheses for selected cases; to optimize crystal size; to establish exact polymerization conversions; to establish analytical tools for routine 2DP analysis [HR-TEM, Raman-mapping, molecular scale AFM and STM (where appropriate), etc.]; to standardize exfoliation procedure and determine exfoliation yield; to obtain stable single sheet dispersions for polymer physical studies; to investigate sheet properties e.g. the in-plane elastic modulus; to compare synthetic 2DPs with natural 2DPs such as graphene.

Coming to the end, a recent result from an exfoliation study involving the above irradiated crystals shall be mentioned. Dr.

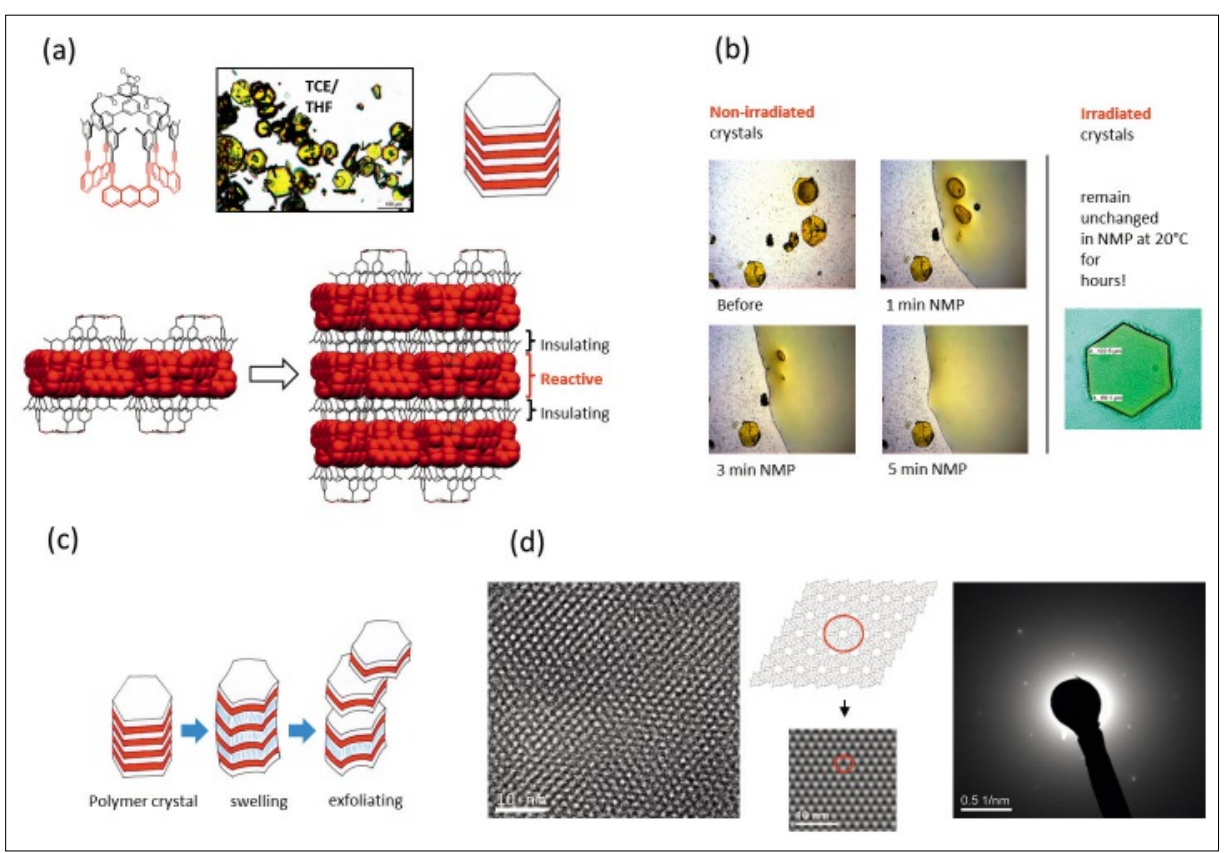

Fig. 6. Converting a monomer single crystal into a 2DP. (a) Chemical structure of monomer. Optical micrograph (OM) of its single crystals. Internal packing into layers. (b) OM images of monomer crystals which dissolve in N-methylpyrrolidone (NMP) contrasted with an irradiated crystal (false colors) which does not dissolve anymore under the same conditions. (c) Sketch of the exfoliation process. (d) Wiener-filtered TEM image of an irradiated crystal, a model explaining the contrast and an electron diffractogram proving the order.

Andri Schütz, a postdoc in our group, is presently investigating how to perform exfoliations such that a maximum of single sheets can be obtained. This turned out to be a rather challenging nano-engineering matter and it is still not clear whether a fully satisfactory solution can eventually be found. Fig. 7 shows an overview scanning electron microscopy (SEM) image of the transient situation, currently attained by Andri. Irradiated crystals (irradiation length is important) were subjected to microwave heating (heating/cooling profile and applied temperature are important) for a certain time (time is important) and the obtained dispersions (difficult to determine quantitatively how many crystals have disappeared) filtered through a TEM $\mathrm{Cu}$-grid, the holes of which were covered with a holey network of amorphous carbon
(Lacey carbon). Without this network all sheets would simply pass through the grid holes and the filtering effect would be zero. But even with this arrangement it cannot be assured that single sheets, which are expected to be rather flexible, do not pass through the much, much smaller pores of the carbon network. Despite these complications the SEM image is remarkable in that it shows many, many features in a size range that reasonably corresponds to the crystals used for exfoliation. Also the features, which are believed to be sheet packages, are more or less transparent which means that they comprise of a few up to $\sim 50$ sheets. Note that the features burn away in the electron beam the easier the thinner they are. Single sheets do so within a matter of seconds. This can be used as a qualitative criterion to differentiate single

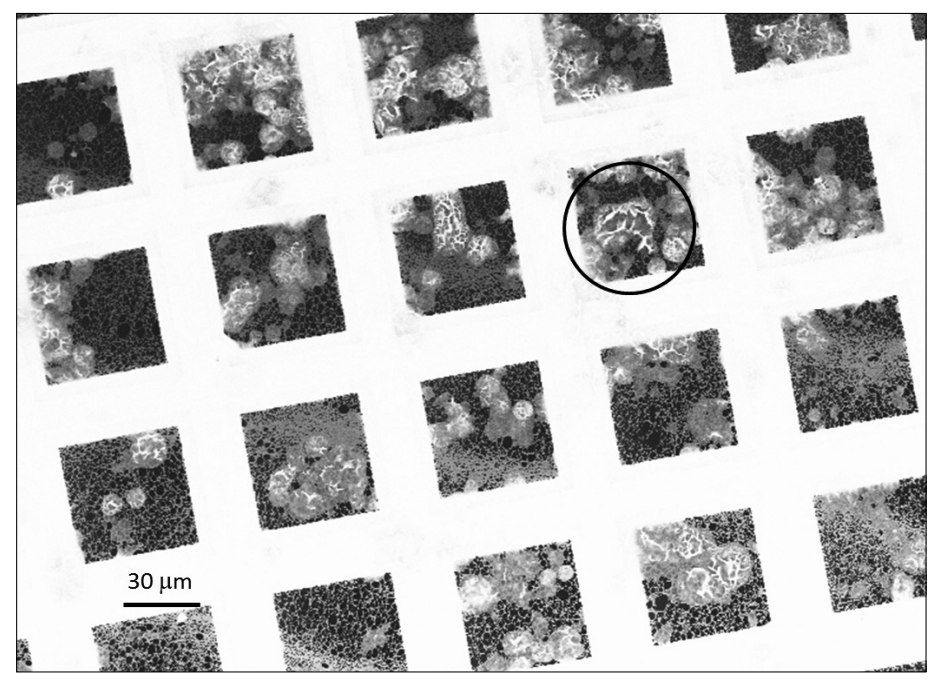

Fig. 7. SEM overview image of 2DP sheets and sheet packages captured on a filter composed of a TEM grid spanned with a fine but irregular network of amorphous carbon (Lacey). A feature with crumpled top is circled. 
sheets from packages. Many of the packages exhibit a structure on their top reminiscent of gathering a tablecloth with one's hand. This is considered an indication of the top layers being caught while undergoing further exfoliation.

\section{Summary and Outlook}

Polymer chemistry is an exciting and creative field of research. It allows putting fantasy and imagination into reality and eventually societal use. Once target structures have been identified the whole repertoire of organic and polymer synthesis combined with the analytical arsenal available these days is at one's disposal and everything has just to be combined in the proper way to achieve them. The two projects presented here were started 1992 (DPs) and 2002 (2DP), which is mentioned to make clear that without stamina exploring new territory may not lead anywhere. The author considers the present trend for short financing schemes and demand for immediate success (whatever this is) detrimental to progress in science. Breakthroughs require a mental, financial and operational environment without restrictions, deadlines and the like. Also it is not considered helpful to evaluate scientific performance by bibliometric indices and other questionable measures such as the h-index because this may discourage people to go for risky, long-term goals. Both projects have now reached the state in which they have gained their own momentum. Experiencing this transition from the period in which one always has to push and push in order to keep a project going to the period in which the same runs all by itself is a wonderful and rewarding moment making one forget about all the trouble and frustration one had to go through.

In regard to DPs, synthesis has reached the dimensions of smaller biological functional units and it may well be in future that such units can be mimicked not only in their size but also in their function. Obviously, macromolecules that should serve such purposes need to be far more intelligently designed than the present, rather primitive cases. Additionally, it will be an important future goal to use the divergent growth method to generate molecular colloidal particles. This will open up access to as yet unexplored size ranges which so far were inaccessible for single molecules and also make available soft matter nano-sized particles with an unheard level of internal structure control.

Regarding the 2DP project a critical contemporary will point to its present narrowness. Having just one proven case is in fact a severe weakness which has to be worked on with all energy. It is therefore noted that two further crystal-based 2DPs are about to be published and also the interfacial approach is coming along very well. Thus, it seems as if a gold mine was discovered providing access to sheets with structurally different RUs under ambient conditions allowing for rational organic synthesis.

\section{Acknowledgements}

This work was only possible because of the engaged work and creative input by my coworkers and colleagues. The names not (yet) contained in the publications are Chiara Gstrein (DP), Wenyang Dai, Payam Payamyar, Marco Servalli, and Dr. Zhikun Zheng (2DP). Particulary important contributions came from Prof. Afang Zhang and Dr. Baozhong Zhang in the field of thick polymers and from Priv. Doz. Dr. Junji Sakamoto and Dr. Patrick Kissel in the field of 2DP. Both activities were further prominently supported by Profs. Avraham Halperin, Grenoble, and Martin Kröger, ETHZ, as well as em. Prof. Gerhard Wegner, MPI-P, Mainz. A fruitful collaboration on 2DP with Prof. Benjamin T. King, UN Reno, is also gratefully acknowledged. We rely on a network of specialists who help us with structure and property analysis. This includes Drs. and Profs. Carlos Aleman, TU Barcelona, Michal Borkovec, U Geneva, Paola Ceroni, U Bologna, Rolf Erni, Empa Dübendorf, Lay-Theng Lee, CEA Saclay, Jean-Christophe Leroux, ETHZ, Raffaele Mezzenga, ETHZ, Antonella Rossi, ETHZ, Manfred Schmidt, U Mainz, Andreas Stemmer, IBM/ETHZ, Motomu Tanaka, U Heidelberg, Joost VanderVondele, ETHZ, Dimitris Vlassopoulos, FORTH Heraklion, Renato Zenobi, ETHZ, and all their involved coworkers without whom we would not be where we are! I would like to cordially thank all these supporters for their continued interest, support and encouragement. The author thanks Profs. Ivan Gitsov, SUNY-ESF, and Paul Smith, ETHZ, for helpful comments to the article. Financial support came from the Swiss National Science Foundation (SNF) and through ETH grants which is most gratefully acknowledged.

\section{Received: July 4, 2013}

[1] H. Staudinger, Ber. Dtsch. Chem. Ges. 1920, 53, 1073; H. Staudinger, J. Fritschi, Helv. Chim. Acta 1922, 5, 785.

[2] H. F. Mark, Naturwiss. 1980, 67, 477; H. Ringsdorf, Angew. Chem., Int. Ed. 2004, 43, 1064.

[3] L. Resconi, L. Cavallo, A. Fait, F. Piemontesi, Chem. Rev. 2000, 100, 1253; G. Wilke, Angew. Chem., Int. Ed. 2003, 42, 5000; L. L. Böhm, Angew. Chem., Int. Ed. 2003, 42, 5010; P. D. Hustad, Science 2009, 325, 704.

[4] http://plastics.americanchemistry.com/ Automotive

[5] For example, see: K. Matyjaszewski, J. Xia, Chem. Rev. 2001, 101, 2921.

[6] D. Neher, Macromol. Rapid Commun. 2001, 22, 1365; U. Scherf, E. J. W. List, Adv. Mater. 2002, 14, 477; O. Inganas, F. Zhang, M. R. Andersson, Acc. Chem. Res. 2009, 42, 1731 .

[7] J. Sakamoto, M. Rehahn, G. Wegner, A. D. Schlüter, Macromol. Rapid Commun. 2009, 30, 653.

[8] S. Kwolek, US patent RE30,352, July 29, 1980.

[9] P. Smith, P. J. Lemstra, J. Mater. Sci. 1980, 15, 505.
[10] O. Bertran, B. Zhang, A. D. Schlüter, A. Halperin, M. Kröger, C. Aleman, RSC $A d v$. 2013, 3, 126; O. Bertran, B. Zhang, A. D. Schlüter, A. Halperin, M. Kröger, C. Aleman, J. Phys. Chem. B 2013, 117, 6007 .

[11] B. Zhang, R. Wepf, K. Fischer, M. Schmidt, S. Besse, P. Lindner, B. T. King, R. Sigel, P. Schurtenberger, Y. Talmon, Y. Ding, M. Kröger, A. Halperin, A. D. Schlüter, Angew. Chem., Int. Ed. 2011, 50, 737.

[12] J. Sakamoto, J. Van Heijst, O. Lukin, A. D. Schlüter, Angew. Chem., Int. Ed. 2009, 48, 1030.

[13] E. Buhleier, W. Wehner, F. Vögtle, Synthesis 1978, 155.

[14] D. A. Tomalia, A. M. Naylor, W. A. Goddard III, Angew. Chem., Int. Ed. 1990, 29, 138.

[15] M.-L. Lartigue, B. Donnadieu, C. Galliot, A.-M. Caminade, J.-P. Majoral, J.-P. Fayet, Macromolecules 1997, 30, 7335. Also, see: J. Lim, M. Kostiainen, J. Maly, V. C. P. da Costa, O. Annunziata, G. M. Pavan, E. E. Simanek, J. Am. Chem. Soc. 2013, 135, 4660.

[16] R. Freudenberger, W. Claussen, A. D. Schlüter, H. Wallmeier, Polymer 1994, 35, 4497.

[17] L. Shu, I. Gössl, J. P. Rabe, A. D. Schlüter, Macromol. Chem. Phys. 2002, 203, 2540; R. AlHellani, A. D. Schlüter, Helv. Chim. Acta 2006, 89, 2745; R. Al-Hellani, J. Barner, J. P. Rabe, A. D. Schlüter, Chem. Eur. J. 2006, 12, 6542; Y. Guo, J. D. Van Beek, B. Zhang, M. Colussi, P. Walde, A. Zhang. M. Kröger, A. Halperin, A. D. Schlüter, J. Am. Chem. Soc. 2009, 131, 11841. Also, see: B. Zhang, H. Yu, A. D. Schlüter, A. Halperin, M. Kröger, Nat. Commun. 2013, in press. DOI: $10.1038 /$ ncomms 2993.

[18] P. G. De Gennes, H. Hervet, J. Phys. Lett. 1983, 44, L351.

[19] B. Zhang, R. Wepf, M. Kröger, A. Halperin, A D. Schlüter, Macromolecules 2011, 44, 6785.

[20] B. Zhang, A. Kröger, C. Rosenauer, A. D. Schlüter, G. Wegner, Coll. Polym. Sci. 2013, accepted.

[21] D. Kurzbach, D. R. Kattnig, B. Zhang, A. D Schlüter, D. Hinderberger, Chem. Sci. 2012, 3, 2550 .

[22] A. Meller, Adv. Chem. Phys. 2012, 149, 251; A. Oukhaled, L. Bacri, M. Pastoriza-Gallego, J.M. Betton, J. Pelta, ACS Chem. Biol. 2012, 7, 1935.

[23] I. Popa, B. Zhang, P. Maroni, A. D. Schlüter, M. Borkovec, Angew. Chem., Int. Ed. 2010, 49, 4250.

[24] M. Kröger, A. D. Schlüter, A. Halperin, Macromolecules 2013, submitted.

[25] M. R. Falvo, R. M. Taylor II, A. Helser, V. Chi, F. P. Brooks Jr., S. Washburn, R. Superfine, Nature 1999, 397, 236.

[26] J. C. Daniel, R. Audebert, 'Small Volumes and Large Surfaces: The World of Colloids', in: 'Soft Matter Physics', Eds. M. Daoud, C. E. Williams, Springer, Berlin/Heidelberg, 1999.

[27] J. W. Colson, W. R. Dichtel, Nat. Chem. 2013, advanced online.

[28] There are a few claims of having achieved exfoliations down to the single layer sheet without, however, providing a mass balance: J.-C. Tan, P. J. Saines, E. G. Bithell, A. K. Cheetham, ACS Nano 2012, 6, 615; A. Gallego, C. Hermosa, O. Castillo, I. Berlanga, C. J. Gómez-García, E. Mateo-Martí, J. I. Martínez, F. Flores, C. Gómez-Navarro, J. GómezHerrero, S. Delgado, F. Zamora, Adv. Mat. 2013, 25, 2141

[29] T. Bauer, Z. Zheng, A. Renn, R. Enning, A. Stemmer, J. Sakamoto, A. D. Schlüter, Angew. Chem., Int. Ed. 2011, 50, 7879.

[30] P. Kissel, R. Erni, W. B. Schweizer, M. D. Rossell, B. T. King, T. Bauer, S. Götzinger, A D. Schlüter, J. Sakamoto, Nat. Chem. 2012 4, 287; R. Bhola, P. Payamyar, D. J. Murray, B. Kumar, A. J. Teator, M. U. Schmidt, S. Hammer, A. Saha, J. Sakamoto, A. D. Schlüter, B. T. King, J. Am. Chem. Soc. 2013, 135, 14134.

[31] G. M. J. Schmidt, 'Solid State Photochemistry', Verlag Chemie, Weinheim, Germany, 1976.

[32] J. N. Coleman, Acc. Chem. Res. 2013, 46, 14. 